\title{
Risk Perception, Self-efficacy, Lay Theories of Health, and Engagement in Health-Protective Behaviors Among Hospital Pharmacists During the COVID-19 Pandemic
}

\author{
Ning Zhang ${ }^{1} \cdot$ Hongyu Yang ${ }^{2} \cdot$ Dongsheng Hong ${ }^{2} \cdot$ Xin Huang $^{2} \cdot$ Linrun Wang $^{2}$
}

Accepted: 24 May 2021 / Published online: 23 June 2021

(c) International Society of Behavioral Medicine 2021

\begin{abstract}
Background Risk perception is an important predictor of health-protective behaviors during pandemics. However, the underlying mechanism connecting risk perception and health-protective behaviors is not well understood. The current study investigates how risk perception predicts hospital pharmacists' engagement in health-protective behaviors during the peak period of COVID-19 pandemic in China and the mediating effects of lay theories of health and self-efficacy.

Method A cross-sectional study on risk perception and engagement in health-protective behaviors was conducted among hospital pharmacists during the COVID-19 pandemic in China. A total of 4121 hospital pharmacists completed the study. Results Risk perception, self-efficacy, and lay theories of health were significant predictors of health-protective behaviors among pharmacists. Lay (entity) theories of health and self-efficacy mediated the relationship between risk perception and engagement in health-protective behaviors among hospital pharmacists.

Conclusion Risk perception, self-efficacy, and lay theories (entity versus incremental) of health significantly predicted hospital pharmacists' engagement in health-protective behaviors during the COVID-19 pandemic in China.
\end{abstract}

Keywords Risk perception $\cdot$ Self-efficacy $\cdot$ Lay theories of health $\cdot$ Health-protective behaviors $\cdot$ COVID-19

\section{Introduction}

The COVID-19 pandemic is the most severe emergent infectious disease pandemic in the twenty-first century. As there are no effective antiviral drugs to contain the pandemic and it

\author{
Linrun Wang \\ linrunw@zju.edu.cn \\ Ning Zhang \\ zhangning2019@zju.edu.cn \\ Hongyu Yang \\ hongyuyang@zju.edu.cn \\ Dongsheng Hong \\ hdswell@zju.edu.cn \\ Xin Huang \\ huangxin3626@zju.edu.cn
}

1 School of Public Health and the Second Affiliated Hospital of Zhejiang University School of Medicine, 310058 Hangzhou, China

2 Department of Pharmacy, the First Affiliated Hospital, Zhejiang University School of Medicine, Hangzhou 310003, China would take much longer than expected to achieve herd immunity through vaccination, engagement in health-protective behaviors such as wearing face masks and social distancing is pivotal to contain the COVID-19 pandemic [1-5]. Previous research demonstrated that risk perception significantly predicted people's responses to pandemics of infectious diseases such that those who perceived a higher level of risk were more likely to engage in health-protective behaviors [6-9]. For instance, Rubin and colleagues found that perceived severity of the swine flu was associated with recommended behavior change in UK [7]. Recent research on risk perception during the COVID-19 pandemic across Asia, Europe, and the USA also found that risk perception was significantly correlated with self-reported adoption of preventative health behaviors [2, 10]. Previous research during pandemics such as 2002-2003 SARS, 2009 H1N1 influenza, however, found that initiating and implementing health-protective behaviors was a big challenge to contain infectious diseases [11]. Even when people are motivated to protect themselves and others from infection, they may not always engage in the recommended health-protective behaviors, and therefore, reduce the efficiency of coordinated efforts to contain the COVID-19 pandemic. Therefore, it is 
important to understand and underlying mechanisms that influence people's likelihood of engaging in health-protective behaviors to inform best practices for improving infection control and enhancing pandemic preparedness [10].

Given that population-wide engagement in health-protective behaviors is critical for reducing the transmission of infectious diseases [3-5], more research is needed to understand the mechanisms that accounts for people's engagement in healthprotective behaviors. As many pharmacists actively engaged in the coordinated efforts to contain the COVID-19 pandemic, such as developing local treatment protocols and participating in patient rounds, they faced a high risk of infection during the pandemic [12-14]. Therefore, it is important to understand the factors that influence their engagement in health-protective behaviors during COVID-19.

Inspired by the research on lay theories by Dweck and colleagues [15-17], one psychological mechanism that draws increasing attention among health psychologists in predicting health behavior is people's lay theories of health-the naïve understanding that people believe their health is malleable through their own behavior (incremental theory) or determined by uncontrollable factors (entity theory). For example, Bunda and Bursseri found that inducing incremental versus entity theory of health through experiments could enhance people's intentions to engage in health-promoting behaviors (e.g., exercising) [18]. Recent research also demonstrated that implicit theories of health predicted people's engagement in health-protective behaviors through enhancing their likelihood of considering future consequences [19].

\section{The Current Research}

The current research investigates the role of lay theories of health in explaining pharmacists' likelihood of engaging health-protective behaviors during the COVID-19 pandemic in China. As previous research demonstrated that self-efficacy predicted health behaviors such that those held a higher level of self-efficacy were more likely to engage in health-protective behaviors [20,21], we also included self-efficacy of protecting oneself from infection during the COVID-19 pandemic as a predictor. We hypothesized that lay theories of health and selfefficacy would mediate the relationship between risk perception and engagement in health-protective behaviors among hospital pharmacists during the COVID-19 pandemic in China.

\section{Methods}

\section{Participants}

A total of 4219 hospital pharmacists completed the study through an online survey platform (www.wjx.com) shared among working groups of pharmacists across China in middle February, 2020. They consented to participate in the study upon opening the survey link and did not receive any financial incentives for participation. Based on priori recruiting criteria decided before initiation of this study, 98 participants were excluded from the final analysis: two participants reported extreme age values, and 96 participants took less than $240 \mathrm{~s}$ or more than $7200 \mathrm{~s}$ to finish the survey which were viewed as not taking the study seriously. Most participants are female $(75.8 \%)$, which is in line with the gender distribution of pharmacists in China [22]. The age of participants ranges from 20 to 73 years old $\left(M_{a g e}=35.96\right.$ years, $S D=8.25)$. Most of the participants are married $(76.4 \%)$ and have a bachelor's degree or above (87.8\%).

\section{Measures and Procedures}

Risk Perception and Self-Efficacy Participants' risk perception of the COVID-19 pandemic was measured with four items and self-efficacy with two items adapted from previous research on risk perception and self-efficacy during pandemics [23]. The reliability for risk perception and self-efficacy was $\alpha=0.64$ and $\alpha=0.87$, respectively. Both the risk perception and self-efficacy measure has been translated into Chinese and verified for use in China before [23].

Health-Protective Behaviors Adapted from previous research on health-protective behaviors during the SARS pandemic [24-26], we measured health-protective behavior during the COVID-19 with 15 items (e.g., "wearing a face mask while going out"). The reliability of health-protective behaviors is high (Cronbach's $\alpha=0.87$ ).

Lay Theories of Health Lay theories of health were measured by the Lay Theory of Health Measure [18]. The scale measures entity versus incremental theory of health with six items. The measured was obtained from the original researcher and translated by two Chinese-English bilinguals using the translation and back-translation method [27]. The reliability for the lay theories of health measure in the current study is satisfactory (Cronbach's $\alpha=0.80$ ). Exploratory factor analysis with varimax rotation and maximum likelihood extraction method verified the two-factor structure of the lay theories of health scale, with factor loadings higher than 0.60 for all items in the current study. The items of the measures used in this study are presented in the online supplementary material.

Finally, participants finished demographic questions on age, gender, education level, marital status, whether they have child(ren) or not, unit of pharmacy they worked in, length of working experience, and whether the hospital 
they worked with had adequate protective measures during COVID-19. This study received ethic clearance from Research Ethics Committee of the authors' hosted institution.

\section{Data Analysis}

All analyses were conducted using SPSS 22 software. We first reported descriptive statistics on risk perception, selfefficacy, lay theories of health, and frequency of engaging in health-protective behaviors among hospital pharmacists. A correlation matrix was created to describe the correlations between key variables included in this study. Multiple regression analyses were conducted to explore the predictors of engagement in health-protective behaviors among hospital pharmacists. Finally, we conducted mediational analyses to investigate whether lay theories (incremental versus entity) of health and self-efficacy mediate the relationship between risk perception and engagement in health-protective behaviors using the indirect macro developed by Preacher and Hayes [28].

\section{Results}

Descriptive characteristics of the sample are reported in Table 1 of the supplementary material. Correlational analyses revealed that risk perception negatively correlated with self-efficacy in preventing themselves from infection with COVID-19, self-efficacy positively correlated with incremental theory of health and frequency of engaging in healthprotective behaviors, but negatively correlated with entity theory of health, incremental theory of health positively correlated with engagement in health-protective behaviors, and entity theory of health negatively correlated with engagement in health-protective behaviors (see Table 2 of the supplementary material).

Multiple Regressions Regression analyses revealed that age, length of working experience, whether the hospital had adequate protective measures, risk perception, self-efficacy, and incremental and entity theory of health were significant predictors for pharmacists' engagement in health-protective behaviors during COVID-19. These results suggested that pharmacists who had longer working experiences engaged in health-protective behaviors more frequently. However, older pharmacists reported a lower level of engagement in healthprotective behaviors. Those perceived a higher level of risk, had a higher level of self-efficacy in preventing themselves from infection, and a higher level of incremental theory of health and a lower level of entity theory of health were more likely to engage in health-protective behaviors during COVID-19.

Mediational Analyses Mediation analysis revealed that the indirect effect of risk perception on engagement in healthprotective behaviors through lay theories of health was
Table 1 Multiple regressions of effect of risk perception on health-protective behaviors

\begin{tabular}{|c|c|c|c|c|c|c|}
\hline \multirow{4}{*}{ Variables } & \multirow{2}{*}{\multicolumn{2}{|c|}{$\frac{\text { Model } 1}{(N=4121)}$}} & \multirow{2}{*}{\multicolumn{2}{|c|}{$\frac{\text { Model } 2}{(N=4121)}$}} & \multirow{2}{*}{\multicolumn{2}{|c|}{$\frac{\text { Model } 3}{(N=4121)}$}} \\
\hline & & & & & & \\
\hline & \multicolumn{2}{|l|}{ HPB } & \multicolumn{2}{|l|}{ HPB } & \multicolumn{2}{|l|}{ HPB } \\
\hline & $\beta$ & $p$ & $\beta$ & $p$ & $\beta$ & $p$ \\
\hline \multicolumn{7}{|l|}{ 1: Control variable } \\
\hline Age & -0.097 & $<.001$ & -0.116 & $<.001$ & -0.094 & $<.001$ \\
\hline Gender & 0.070 & $<.001$ & 0.090 & $<.001$ & 0.093 & $<.001$ \\
\hline Education & -0.019 & .225 & -0.017 & .272 & -0.018 & .217 \\
\hline Working experience (ys) & 0.066 & .004 & 0.065 & .003 & 0.060 & .005 \\
\hline Protective measure adequacy & -0.148 & $<.001$ & -0.099 & $<.001$ & -0.096 & $<.001$ \\
\hline \multicolumn{7}{|l|}{ 2: Independent variables } \\
\hline Risk perception & & & 0.086 & $<.001$ & 0.086 & $<.001$ \\
\hline Self-efficacy & & & 0.229 & $<.001$ & 0.188 & $<.001$ \\
\hline \multicolumn{7}{|l|}{ 3: Mediator } \\
\hline Incremental theory of health & & & & & 0.096 & $<.001$ \\
\hline Entity theory of health & & & & & -0.196 & $<.001$ \\
\hline$R^{2}$ & 0.032 & & 0.08 & & 0.129 & \\
\hline$\Delta F$ & $26.778 * * *$ & & $108.658^{* * *}$ & & $114.998 * * *$ & \\
\hline$\Delta R^{2}$ & & & 0.049 & & 0.049 & \\
\hline
\end{tabular}

$H P B$ Health-protective behaviors, ${ }^{* * *} p<.001$ 
Table 2 Indirect effect of on risk perception on HPB

\begin{tabular}{lcllll}
\hline Paths & Effect & BootSE & $t$ & $p$ & $\begin{array}{l}\text { Bootstrapping } \\
\text { bias-corrected } \\
\end{array}$ \\
& & & & & $95 \%$ CI \\
\hline Total effect $\left(\mathrm{X}_{1} \rightarrow \mathrm{Y}_{1}\right)$ & 0.047 & 0.015 & 3.174 & $0.002^{* *}$ & $(0.018,0.076)$ \\
Direct effect & 0.084 & 0.014 & 5.886 & $0.000^{* * *}$ & $(0.056,0.112)$ \\
Indirect effect(s) & & & & & $(-0.048,-0.027)$ \\
Total & -0.037 & 0.005 & & & $(-0.004,0.002)$ \\
Ind1 $\left(\mathrm{X}_{1} \rightarrow \mathrm{M}_{1} \rightarrow \mathrm{Y}_{1}\right)$ & -0.001 & 0.001 & & & $(-0.013,0.000)$ \\
Ind2 $\left(\mathrm{X}_{1} \rightarrow \mathrm{M}_{2} \rightarrow \mathrm{Y}_{1}\right)$ & -0.006 & 0.003 & & & $(-0.038,-0.023)$ \\
Ind3 $\left(\mathrm{X}_{1} \rightarrow \mathrm{M}_{3} \rightarrow \mathrm{Y}_{1}\right)$ & -0.030 & 0.004 & & & \\
\hline
\end{tabular}

Confidence intervals $(\mathrm{CI})$ not including zero demonstrate a statistically significant effect

$X_{1}$ risk perception, $Y_{1}$ health-protective behaviors, $M_{1}$ incremental theory of health, $M_{2}$ entity theory of health, $M_{3}$ self-efficacy

${ }^{* *} p<0.01 ; * * * p<0.001$ significant, $B=-0.011, p<0.001$. Bootstrapping analysis revealed that the $95 \%$ bias-corrected CI $[-0.020,-0.004]$ did not include zero. Another mediation analysis with entity versus incremental theory of health entered into the model as two separate mediators between risk perception and engagement in health-protective behaviors revealed that the model was significant, $B=-0.011, p<0.001$. Bootstrapping analyses revealed that the $95 \%$ bias-corrected CI for incremental theory of health $[-0.006,0.002]$ included zero; however, the $95 \%$ bias-corrected CI for entity theory of health $[-0.016,-0.001]$ did not include zero.

When self-efficacy in preventing oneself from infection was included as a mediator simultaneously with lay theories of health, the results revealed that both entity theory of health and self-efficacy mediated the impact of risk perception on engagement in health-protective behaviors among pharmacists, bootstrapping analyses revealed that the $95 \%$ bias-corrected CI for entity theory of health $[-0.013,0.000]$ and self-efficacy $[-0.038,-0.023]$ did not include zero, and the $95 \%$ biascorrected CI for incremental theory of health [-0.004, 0.002] included zero. Therefore, the entity theory of health and selfefficacy mediated the effect of risk perception on pharmacists' engagement in health-protective behaviors during the COVID19 pandemic (see Table 2).

\section{Discussion}

This study investigated risk perception, self-efficacy, lay theories of health, and frequency of engaging in health-protective behaviors among hospital pharmacists during the COVID19 pandemic in China. As expected, correlational analyses revealed that pharmacists' self-efficacy and incremental theory of health were positively correlated with their engagement in health-protective behaviors during the pandemic whereas entity theory of health was negatively correlated with engagement in health-protective behaviors. Regression analyses confirmed the predicting effect of self-efficacy and incremental and entity theory of health on pharmacists' engagement in health-protective behaviors during COVID-19. These results highlighted the value of including self-efficacy and lay theories (incremental versus entity) of health in understanding people's likelihood of engaging in health-protective behaviors during pandemics of infectious diseases [19].

The current research contributes to our understanding on the psychological mechanisms of risk perception on healthprotective behaviors such that pharmacists' lay theories of health mediated the relationship between their risk perception of the pandemic and engagement in health-protective behaviors. Previous research has found that incremental theory of health predicted people's likelihood of engaging in healthpromoting behaviors such as engaging in healthy eating and exercising and maintaining a healthy weight $[18,29]$. The current research demonstrated that risk perception could have a dampening effect on engagement in health-protective behaviors through increasing entity theory of health. The results have implications for risk communication during the pandemic such that strategies to help people calibrate their perceived risk of infection could insulate their lay theories of health from the dampening effect of risk perception, and in turn, enhance engagement in health-protective behaviors.

The results also have practical implications for developing effective, applicable, and scalable interventions to promote health-protective behaviors during the pandemic through reducing the entity theory of health or increasing the incremental theory of health and self-efficacy. Previous research has already demonstrated the effectiveness of inducing incremental theory of health through experimental methods [18, 29]; future research can test the effectiveness of interventions to increase incremental theory of health in promoting health-protective behaviors during pandemics of infectious diseases. Future research can also focus on developing scalable public health interventions to enhance 
incremental theory of health and self-efficacy among the general public to promote health-protective behaviors and enhance pandemic preparedness, especially among countries with low engagement in health-protective behaviors [30].

The current study also has several limitations. First, the results of the current study are correlational rather than causal. Future research can test the effectiveness of brief interventions to foster incremental theory of health in promoting health-protective behaviors. Second, engagement in health-protective behaviors was measured through selfreport which could be biased by cognitive and motivational factors; future research should incorporate more objective measures on health-protective behaviors [31]. Lastly, as the participants in the study are hospital pharmacists, the results may not be generalizable to other healthcare professionals or the general population. Future research is warranted to increase the generalizability of the current research results.

In conclusion, the current research demonstrated that lay theories (entity versus incremental) of health mediated the relationship between risk perception and frequency of engaging in health-protective behaviors among hospital pharmacists during the COVID-19 pandemic in China. Reducing the entity theory of health and fostering the incremental theory of health could be effective, applicable, and scalable strategies to reduce the motivational barriers of behavioral change during public health crisis like COVID-19 and to promote engagement in health-protective behaviors among the general public to improve pandemic preparedness.

Supplementary Information The online version contains supplementary material available at https://doi.org/10.1007/s12529-021-10004-2.

Acknowledgements The authors would like to thank Mengxi Guo for her help with statistical analyses in revising the manuscript. They also would like to thank the anonymous reviewers and the editors of International Journal of Behavioral Medicine for their constructive comments in revising the manuscript.

Author Contributions N.Z helped designed the research, analyzed the data, and wrote the manuscript and D.S.H, H.Y.Y, X.H, L.R.W helped with data collection and provided comments on revising the manuscript. All the authors agree with the submission of this manuscript for publication.

Funding This study was funded by Hundred Talents Program Research Initiation Fund from Zhejiang University, Zhejiang University special scientific research fund for COVID-19 prevention and control (2020XGZX046), and the Weijian Special Fund from the Zhejiang University Educational Foundation (419000-11143/017).

\section{Declarations}

Ethical Approval All procedures performed in studies involving human participants were in accordance with the ethical standards of the institutional research committee and with the 1964 Helsinki declaration and its later amendments or comparable ethical standards.
Conflict of Interest The authors declare that they have no conflict of interest.

\section{References}

1. Iorfa SK, Ottu IF, Oguntayo R, et al. COVID-19 Knowledge, risk perception, and precautionary behavior among Nigerians: a moderated mediation approach. Front. Psychol. 2020;11.

2. Wise T, Zbozinek TD, Michelini G, Hagan CC, Mobbs D. Changes in risk perception and self-reported protective behaviour during the first week of the COVID-19 pandemic in the United States. R Soc Open Sci. 2020;7(9):200742. https://doi.org/10. 1098/rsos.200742.

3. Michie S, Rubin G, Amlot R. Behavioural science must be at the heart of the public health response to covid-19. BMJ opinion. 2020.

4. West R, Michie S, Rubin GJ, Amlôt R. Applying principles of behaviour change to reduce SARS-CoV-2 transmission. Nat Hum Behav. 2020;4(5):451-9.

5. Zhang N. Behavioral insights for containing the COVID-19 pandemic: some practices in China. Behav. Sci. Policy.

6. Leppin A, Aro AR. Risk perceptions related to SARS and avian influenza: theoretical foundations of current empirical research. Int J Behav Med. 2009;16(1):7-29.

7. Rubin GJ, Amlôt R, Page L, Wessely S. Public perceptions, anxiety, and behaviour change in relation to the swine flu outbreak: cross sectional telephone survey. BMJ. 2009;339.

8. Rudisill C. How do we handle new health risks? Risk perception, optimism, and behaviors regarding the H1N1 virus. J Risk Res. 2013;16(8):959-80.

9. van der Weerd W, Timmermans DR, Beaujean DJ, Oudhoff J, van Steenbergen JE. Monitoring the level of government trust, risk perception and intention of the general public to adopt protective measures during the influenza A (H1N1) pandemic in the Netherlands. BMC Public Health. 2011;11(1):1-12.

10. Dryhurst S, Schneider CR, Kerr J, et al. Risk perceptions of COVID-19 around the world. J Risk Res. 2020;23(7-8):994-1006.

11. Rubin GJ, Potts H, Michie S. The impact of communications about swine flu (influenza A H1N1v) on public responses to the outbreak: results from 36 national telephone surveys in the UK. Health Technol Assess. 2010;14(34):183-266.

12. Carico R Jr. Pharmacists continue to protect communities during COVID-19. J Am Pharm Assoc. 2020;60(5):660.

13. Grabenstein JD. Pharmacists continue to protect communities during COVID-19. J Am Pharm Assoc. 2020;60(5):661.

14. Li M, Razaki H, Mui V, Rao P, Brocavich S. The pivotal role of pharmacists during the 2019 coronavirus pandemic. J Am Pharm Assoc. 2020;60(6):e73-5.

15. Chiu CY, Hong YY, Dweck CS. Lay dispositionism and implicit theories of personality. J Pers Soc Psychol. 1997;73(1):19.

16. Dweck CS, Chiu CY, Hong YY. Implicit theories and their role in judgments and reactions: a word from two perspectives. Psychol Inq. 1995;6(4):267-85.

17. Dweck CS, Chiu CY, Hong YY. Implicit theories: elaboration and extension of the model. Psychol. Inq. 1995;6(4):322-33.

18. Bunda K, Busseri MA. Lay theories of health, self-rated health, and health behavior intentions. J Health Psychol. 2019;24(7):979-88.

19. Zhang N, Kou Y. Implicit theories of health, consideration of future consequences, and engagement in health protective behaviors during the COVID-19 pandemic in China. J. Health Psychol. May 2021. https://doi.org/10.1177/13591053211017191 
20. Maddux JE, Brawley L, Boykin A. Self-efficacy and healthy behavior. In: Maddux JE, editors. Self-Efficacy, adaptation, and adjustment: Springer; 1995. p. 173-202.

21. Strecher VJ, McEvoy DeVellis B, Becker MH, Rosenstock IM. The role of self-efficacy in achieving health behavior change. Health Educ Q. 1986;13(1):73-92.

22. National Health Commission of China. China Health Statistics Yearbook. 2020. Available from: https://data.cnki.net/area/ Yearbook/Single/N2020020200?z=D09.

23. De Zwart O, Veldhuijzen IK, Elam G, et al. Perceived threat, risk perception, and efficacy beliefs related to SARS and other (emerging) infectious diseases: results of an international survey. Int $\mathbf{J}$ Behav Med. 2009;16(1):30-40.

24. Brug J, Aro AR, Oenema A, De Zwart O, Richardus JH, Bishop GD. SARS risk perception, knowledge, precautions, and information sources, the Netherlands. Emerg Infect Dis. 2004;10(8):1486.

25. Puterman E, Delongis A, Lee-Baggley D, Greenglass E. Coping and health behaviours in times of global health crises: lessons from SARS and West Nile. Glob Public health. 2009;4(1):69-81.

26. Vartti A-M, Oenema A, Schreck M, et al. SARS knowledge, perceptions, and behaviors: a comparison between Finns and the Dutch during the SARS outbreak in 2003. Int J Behav Med. 2009;16(1):41.

27. Brislin RW. Back-translation for cross-cultural research. J Crosscult Psychol. 1970;1(3):185-216.

28. Preacher KJ, Hayes AF. Asymptotic and resampling strategies for assessing and comparing indirect effects in multiple mediator models. Behav Res Method. 2008;40(3):879-91.

29. Burnette JL, Finkel EJ. Buffering against weight gain following dieting setbacks: an implicit theory intervention. J Exp Soc Psychol. 2012;48(3):721-5.

30. Gelfand MJ, Jackson JC, Pan X, et al. The relationship between cultural tightness-looseness and COVID-19 cases and deaths: a global analysis. Lancet Planet. Health. 2021.

31. Weinstein ND. Misleading tests of health behavior theories. Ann Behav Med. 2007;33(1):1-10. https://doi.org/10.1207/ s15324796abm3301_1.

Publisher's Note Springer Nature remains neutral with regard to jurisdictional claims in published maps and institutional affiliations. 